Original Article

\title{
Spatiotemporal expression of RCAN1 and its isoform RCAN1-4 in the mouse hippocampus after pilocarpine-induced status epilepticus
}

\author{
Kyung-Ok Cho ${ }^{1,2}$, Kyoung Hoon Jeong ${ }^{1}$, Jung-Ho Cha ${ }^{3}$, and Seong Yun Kim ${ }^{1, *}$ \\ ${ }^{1}$ Department of Pharmacology, Department of Biomedicine \& Health Sciences, Catholic Neuroscience Institute, College of Medicine, The Catholic University \\ of Korea, ${ }^{2}$ Institute of Aging and Metabolic Diseases, College of Medicine, The Catholic University of Korea, ${ }^{3}$ Department of Anatomy, College of Medicine, The \\ Catholic University of Korea, Seoul 06591, Korea
}

\section{ARTICLE INFO}

Received September 21, 2019

Revised October 17, 2019

Accepted November 22, 2019

*Correspondence

Seong Yun Kim

E-mail: syk@catholic.ac.kr

Key Words

Epilepsy

Hippocampus

Regulator of calcineurin 1

Regulator of calcineurin 1-4

Status epilepticus
ABSTRACT Regulator of calcineurin 1 (RCAN1) can be induced by an intracellular calcium increase and oxidative stress, which are characteristic features of temporal lobe epilepsy. Thus, we investigated the spatiotemporal expression and cellular localization of RCAN1 protein and mRNA in the mouse hippocampus after pilocarpine-induced status epilepticus (SE). Male C57BL/6 mice were given pilocarpine hydrochloride $(280 \mathrm{mg} / \mathrm{kg}$, i.p.) and allowed to develop $2 \mathrm{~h}$ of SE. Then the animals were given diazepam $(10 \mathrm{mg} / \mathrm{kg}$, i.p.) to stop the seizures and sacrificed at $1,3,7,14$, or 28 day after SE. Cresyl violet staining showed that pilocarpine-induced SE resulted in cell death in the CA1 and CA3 subfields of the hippocampus from 3 day after SE. RCAN1 immunoreactivity showed that RCAN1 was mainly expressed in neurons in the shammanipulated hippocampi. At 1 day after SE, RCAN1 expression became detected in hippocampal neuropils. However, RCAN1 signals were markedly enhanced in cells with stellate morphology at 3 and 7 day after SE, which were confirmed to be reactive astrocytes, but not microglia by double immunofluorescence. In addition, realtime reverse transcriptase-polymerase chain reaction showed a significant upregulation of RCAN1 isoform 4 (RCAN1-4) mRNA in the SE-induced hippocampi. Finally, in situ hybridization with immunohistochemistry revealed astrocytic expression of RCAN1-4 after SE. These results demonstrate astrocytic upregulation of RCAN1 and RCAN1-4 in the mouse hippocampus in the acute and subacute phases of epileptogenesis, providing foundational information for the potential role of RCAN1 in reactive astrocytes during epileptogenesis.

\section{INTRODUCTION}

Epilepsy is a common neurological disorder characterized by unprovoked recurrent seizures [1]. Seizures can be derived from uncontrolled, synchronous, and excessive neuronal excitation in any brain region. When seizures begin in the temporal lobe, including the hippocampus and the surrounding area, it can be diagnosed as temporal lobe epilepsy (TLE) [2]. Because TLE is the most common form of adult focal epilepsy [2], hippocampal pathophysiology in TLE has been studied extensively using various animal models [3]. However, essential molecular mechanisms that follow acute seizures and their chronological contributions to disease progression are still not clearly understood. Therefore, more vigorous research investigations are needed to discern how the expression patterns of various molecules are altered after acute seizures. Because the hippocampus undergoes continuous changes after acute seizures until chronic epilepsy has developed, basic information is needed about the molecular alterations that (i) (5) This is an Open Access article distributed under the terms of the Creative Commons Attribution Non-Commercial License, which permits unrestricted non-commercial use, distribution, and reproduction in any medium, provided the original work is properly cited. Copyright $\odot$ Korean J Physiol Pharmacol, pISSN 1226-4512, elSSN 2093-3827
Author contributions: K.O.C. and K.H.J. performed the experiments. J.H.C designed the probes for in situ hybridization. S.Y.K. supervised and coordinated the study. K.O.C. and S.Y.K. wrote the manuscript. 
occur during epileptogenesis to better understand the complicated pathophysiology of TLE.

Regulator of calcineurin 1 (RCAN1) is widely expressed in the brain $[4,5]$. The RCAN1 gene encodes two predominant isoforms, RCAN1-1 and RCAN1-4, depending on the choice of the first exon among the 7 exons in the gene [6]. RCAN1-1 mRNA can generate two different protein isoforms, long (RCAN1-1L) and short (RCAN1-1S) proteins, and RCAN1-4 mRNA translates the RCAN1-4 protein when a calcineurin-regulated promoter is activated by an intracellular calcium increase [7-12]. Perturbations in RCAN1 expression have been reported in many brain diseases, including Down syndrome, Alzheimer's disease, Huntington disease, and ischemic stroke [13-18]. Moreover, previous studies demonstrated an association between RCAN1 and oxidative or calcium stress $[19,20]$, which are the most common pathologic mechanisms in multiple brain diseases. Nonetheless, few efforts have been made to discern the role of RCAN1 in epilepsy, despite ample evidence that RCAN1 expression might be altered after acute seizures because calcium overload and excitotoxicity are characteristic features of epilepsy [21].

Therefore, in the present study, we investigated the spatiotemporal expression pattern of RCAN1 in the mouse hippocampus after acute seizures, including a phenotypic analysis. Moreover, we assessed the temporal hippocampal expression of RCAN1-4 mRNA, the calcium-sensitive isoform, using quantitative reverse transcriptase polymerase chain reaction (qRT-PCR), and we used an in situ hybridization double labeling technique to analyze the cell types. These findings provide basic information about the expression patterns of RCAN1 and RCAN1-4 following acute seizures, encouraging further studies to evaluate the functional roles of RCAN1 and its isoforms in epilepsy.

\section{METHODS}

\section{Pilocarpine-induced status epilepticus (SE)}

Animal experiments were approved by the Ethics Committee of the Catholic University of Korea and were carried out in accordance with the National Institutes of Health Guideline for the Care and Use of Laboratory Animals (NIH Publication No. 80-23, revised 1996). The pilocarpine-induced SE model was generated as previously described [22]. Briefly, mice were given atropine methyl nitrate (3 $\mathrm{mg} / \mathrm{kg}$, i.p.) $30 \mathrm{~min}$ before the injection of pilocarpine hydrochloride ( $280 \mathrm{mg} / \mathrm{kg}$, i.p.). Only animals that had stage 5 generalized tonic-clonic seizures according to the Racine scale were selected for the study [23]. After $2 \mathrm{~h}$ of SE, diazepam (10 $\mathrm{mg} / \mathrm{kg}$, i.p.) was administrated to quell the seizures. For sham-manipulated animals, all the procedures were the same except that saline was injected instead of pilocarpine.

\section{Tissue collection and staining}

At 1, 3, 7, 14, and 28 day after SE (1 day after sham-manipulation), the animals were deeply anesthetized with an overdose of ketamine and xylazine. After performing transcardial perfusion with $4 \%$ paraformaldehyde in $0.1 \mathrm{M}$ phosphate buffer ( $\mathrm{pH}$ 7.4), we removed the mouse brains and cryoprotected them with a $30 \%$ sucrose solution. Then, the brains were rapidly frozen with liquid nitrogen and cut into 20 - $\mu \mathrm{m}$-thick coronal sections using a cryostat microtome. Cresyl violet staining and immunohistochemistry were carried out as previously described $[17,22,24]$. For the immunohistochemistry, we used rabbit anti-RCAN1 (1:200), mouse anti-neuronal nuclei (NeuN, 1:200), mouse anti-glial fibrillary acidic protein (GFAP, 1:400), and rat anti-Ox-42 (1:100).

\section{Quantitative reverse transcriptase polymerase chain reaction}

We quantitatively analyzed RCAN1-4 transcription using a previously described method [17]. Briefly, total RNA was extracted from epileptic or sham-manipulated hippocampi, and qRTPCR was performed with SYBR green using an MX3005P system (Stratagene). The primer sequences for RCAN1-4 and glyceraldehyde-3-phosphate dehydrogenase were 5-GTCTGCCCCGTGAAAAAGCA-3' (forward), 5'-TGGAAGGTGGTGTCCTTGTCA-3' (reverse) and 5'-TCAACAGCAACTCCCACTCTTCCA-3' (forward), 5'-ACCCTGTTGCTGTAGCCGTATTCA-3' (reverse), respectively. The fold change of RCAN1-4 transcription was calculated relative to the level in sham-manipulated animals. The results are presented as the mean \pm standard error of the mean and were analyzed using one-way analysis of variance (ANOVA) and a post-hoc Duncan's test. Differences were assumed to be statistically significant at $\mathrm{p}<0.05$.

\section{In situ hybridization double labeling}

The specific sequence for the RCAN1-4 riboprobe was produced by RT, followed by PCR amplification with primer sets (forward: 5'-CTCTTGCAAAGGAACCTCCA-3', reverse: 5'-GGGGGTGGCATCTTCTACTT-3'). After cloning into the pBluescript II RI Predigested Vector (Agilent Technologies, Waldbronn, Germany), digoxigenin (DIG)-labeled sense and antisense riboprobes for RCAN1-4 were prepared by in vitro transcription using a DIG RNA labeling kit (Boehringer, Mannheim, Germany). Coronal sections from sham-treated and epileptic mice were washed three times with $2 \mathrm{x}$ saline-sodium citrate (SSC) buffer $(0.3 \mathrm{M}$ sodium chloride, $0.03 \mathrm{M}$ sodium citrate, $\mathrm{pH}$ 7.0). Then, prehybridization and hybridization steps were performed at $53^{\circ} \mathrm{C}$ for $2 \mathrm{~h}$ and $16 \mathrm{~h}$, respectively. The prehybridization solution was composed of $4 \mathrm{x}$ SSC, $10 \%$ dextran sulfate, $1 \mathrm{x}$ Denhardt's solution, $50 \%$ formamide, and $100 \mu \mathrm{g} / \mathrm{ml}$ salmon sperm DNA. The hybridization buffer was identical to the prehybridization 
solution except for the addition of $500 \mathrm{ng} / \mathrm{ml}$ of sense or antisense RCAN1-4 riboprobes. On the next day, sections were rinsed with $2 \mathrm{x}$ SSC at room temperature and serially washed with prewarmed 2x SSC for $20 \mathrm{~min}, 0.5 \mathrm{x}$ SSC for $45 \mathrm{~min}$, and $0.2 \mathrm{x}$ SSC for $45 \mathrm{~min}$ at $62^{\circ} \mathrm{C}$. After several washes with $0.1 \mathrm{M}$ Tris-buffered saline $(\mathrm{pH}$ 7.5), the sections were incubated with $10 \%$ normal sheep serum containing $0.1 \%$ Triton $\mathrm{X}-100$ for $1 \mathrm{~h}$ at room temperature and subsequently with biotin-conjugated monoclonal mouse antidigoxin (1:500) overnight at $4^{\circ} \mathrm{C}$. On the next day, after labeling with DyLight 488 Streptavidin (1:50) for $2 \mathrm{~h}$ at room temperature, the sections were incubated with mouse anti-GFAP overnight at $4^{\circ} \mathrm{C}$, followed by incubation with Cy-3-conjugated anti-mouse IgG for $2 \mathrm{~h}$ at room temperature. Finally, the slides were observed under a confocal microscope (LSM 510 Meta; Carl Zeiss Co., Ltd., Oberkochen, Germany).

\section{RESULTS}

\section{Hippocampal cell death after pilocarpine-induced SE}

The hippocampal damage caused by pilocarpine-induced SE was assessed by cresyl violet staining. In the sham-manipulated hippocampi, we found intact cells in the pyramidal cell layer of the hippocampus and the hilus of the dentate gyrus (Fig. 1A, A1, A2, and A3). However, 1 day after pilocarpine-induced SE, pyknotic cells were found in the hilar regions of the dentate gyrus (Fig. 1B, B3). Moreover, from 3 to 28 day after SE, pyramidal cells in the medial CA1 and CA3 subfields of the hippocampus, along with the dentate hilar region, showed a clear pyknotic morphology compared with the sham-treated hippocampi (Fig. 1C-E, C1, and C2), validating our in vivo animal model of SE.

\section{Temporal expression pattern and cellular localization of RCAN1 protein after pilocarpine-induced SE}

In the sham-manipulated hippocampi, RCAN1-positive cells were observed in the neurons of the pyramidal and granule cell layer (Fig. 2A and A1). At 1 day after SE, RCAN1 expression was increased in the neuropils of the hippocampal stratum radiatum and the molecular layer of the dentate gyrus (Fig. 2B and B1). At 3 and 7 day after SE, immunoreactivity to RCAN1 was dramatically induced in cells with the stellate morphology and then gradually decreased from 14 to 28 day after SE (Fig. 2C-F, C1). Neuronal RCAN1 signals in the medial CA1 subfield decreased from 3 day until the last time-point examined, possibly due to neuronal deaths caused by the prolonged seizure activity (Fig. 2C-F). The phenotypic analysis of the RCAN1-expressing cells using double
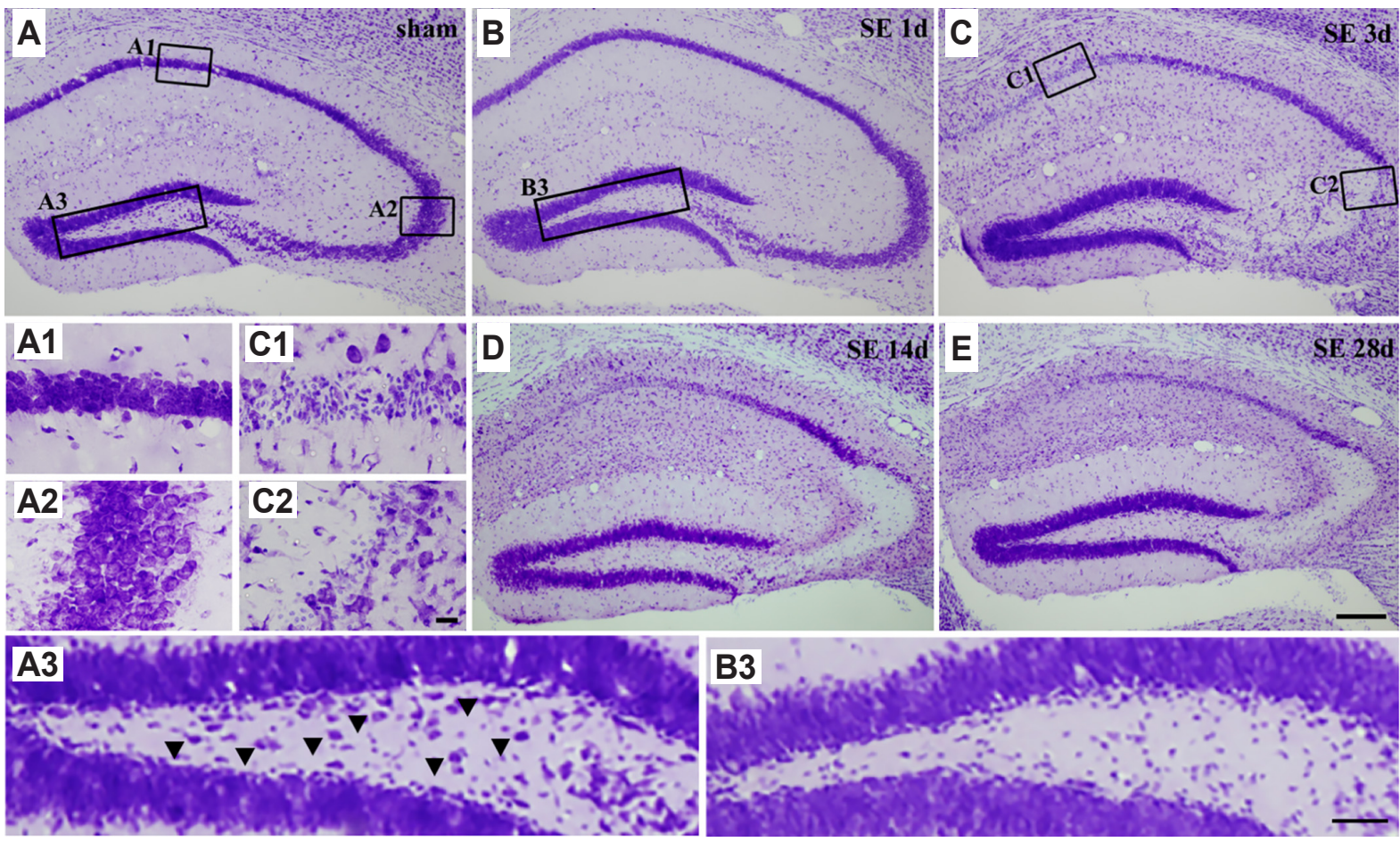

Fig. 1. Hippocampal cell death after pilocarpine-induced status epilepticus (SE) measured by cresyl violet staining. In sham-manipulated hippocampi (A), intact neurons were observed in the CA1 (magnified in A1), CA3 (magnified in A2), and the hilus (magnified in A3, arrowheads for exemplary interneurons) subfields. In contrast, pyknotic cells were found in the hilus 1 day after SE (B, B3), and in the CA1 (magnified in C1) and CA3 (magnified in C2) subfields, along with the dentate hilus at 3 day (C), 14 day (D), and 28 day (E) after SE onset. Scale bar in (E) $=200 \mu \mathrm{m}$; the same magnification was used for (A-D). Scale bar in $(C 2)=20 \mu \mathrm{m}$; the same magnification was used for (A1, A2, C1). Scale bar in (B3) $=50 \mu \mathrm{m}$; the same magnification was used for (A3). 

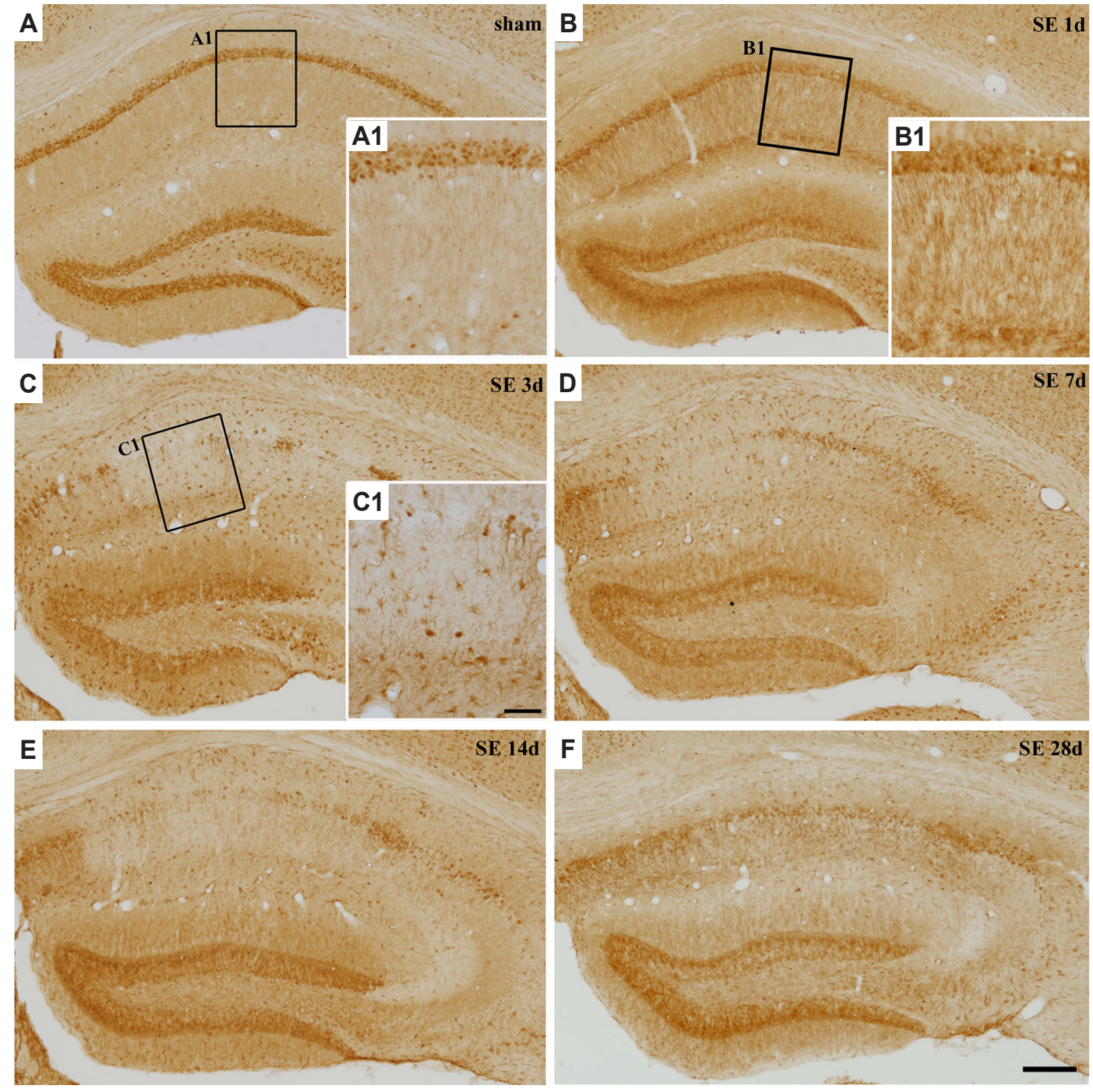

Fig. 2. Temporal expression pattern of regulator of calcineurin 1 (RCAN1) protein in the hippocampus after pilocarpine-induced status epilepticus (SE). In sham-manipulated hippocampi (A), RCAN1 signals were found in neurons (magnified in A1). At 1 day after SE, RCAN1 labeling was increased in neuropils of the hippocampal stratum radiatum (magnified in B1) and the molecular layer of the dentate gyrus (B). RCAN1 expression was markedly induced in glia-like cells at 3 day (C, magnified in C1) and 7 day (D) after SE and gradually subsided from 14 day (E) to 28 day after SE (F). Scale bar in (C1) $=50 \mu \mathrm{m}$; the same magnification was used for (A1, B1). Scale bar in (F) $=200 \mu \mathrm{m}$; the same magnification was used for (A-E).

immunofluorescence revealed that 3 day after SE, RCAN1 in the medial CA1 subfield was co-labeled with a neuronal marker, NeuN (Fig. 3A-C). Moreover, RCAN1 immunoreactivity was colocalized with GFAP, an astrocyte marker (Fig. 3D-F), but not with Ox-42, a microglial marker (Fig. 3G-I).

\section{Temporal expression pattern and cellular localization of RCAN1-4 mRNA after pilocarpine-induced SE}

We further assessed quantitative RCAN1-4 transcription in the hippocampus using qRT-PCR. In agreement with the results of the RCAN1 protein expression analysis, RCAN1-4 mRNA was significantly increased after SE compared with the shammanipulated controls (Fig. 4A). Moreover, cellular localization of RCAN1-4, evaluated by in situ hybridization, showed that in the sham-manipulated hippocampi, RCAN1-4 was observed in the neurons of the pyramidal cell layer and not co-labeled with GFAP, whereas 3 day after SE, the hybridization signal for RCAN1-4 mRNA was localized in astrocytes with GFAP labeling (Fig. 4B, arrows). In addition, no cellular labeling was detected in the hippocampus when in situ hybridization was performed with the RCAN1-4 sense probe (data not shown). 

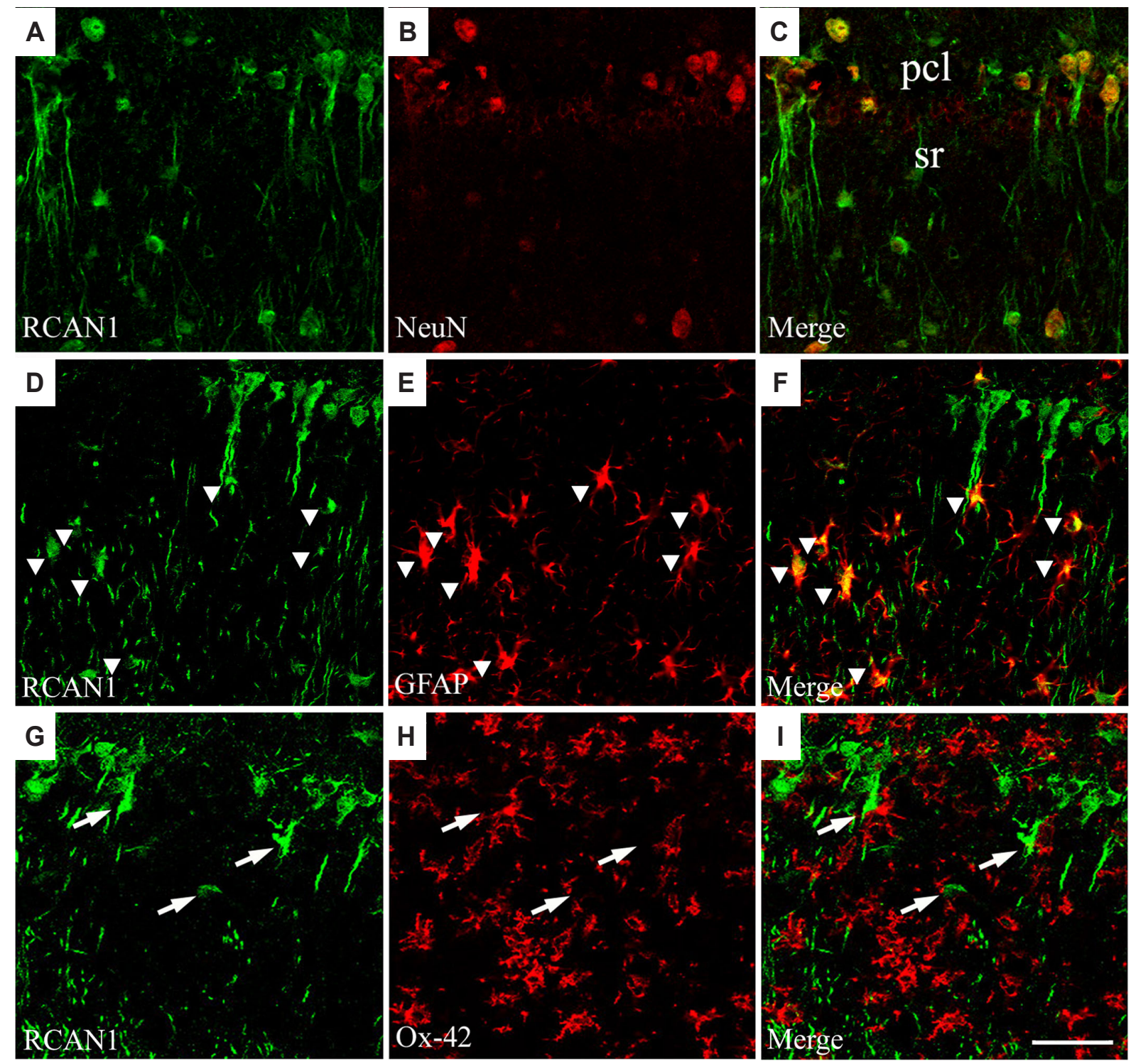

Fig. 3. Phenotypes of regulator of calcineurin 1 (RCAN1)-positive cells in the hippocampus 3 day after pilocarpine-induced status epilepticus (SE). RCAN1 immunoreactivity was co-localized with NeuN (A-C), a neuronal marker, and with GFAP (D-F, arrowheads for RCAN1/GFAP-double positive cells), an astrocyte marker. However, RCAN1 signals did not overlap with Ox-42 labeling (G-I, arrows for RCAN1-positive cells), a microglial marker. Scale bar in $(\mathrm{I})=50 \mu \mathrm{m}$; the same magnification was used for $(\mathrm{A}-\mathrm{H})$. NeuN, neuronal nuclei; pcl, pyramidal cell layer; sr, stratum radiatum; GFAP, glial fibrillary acidic protein.

\section{DISCUSSION}

In the present study, we assessed the spatiotemporal expression pattern of RCAN1 protein and its cellular phenotype in a mouse model of epilepsy. Compared with sham-manipulated controls, RCAN1 expression increased in the neuropils of the hippocampal stratum radiatum and the molecular layer of the dentate gyrus 1 day after SE. At 3 and 7 day after SE, RCAN1 was markedly induced in reactive astrocytes, which gradually decreased from 14 to 28 day after SE. When RCAN1-4, a calcium-inducible RCAN1 isoform, was assessed with qRT-PCR and in situ hybridization double labeling, RCAN1-4 transcription was found to be increased significantly in the hippocampus after acute seizures, in addition to the astrocytic production of RCAN1-4 mRNA, in line with our RCAN1 protein expression data.

A variety of factors, such as exercise, aging, and hyperglycemia, have been reported to stimulate RCAN1 expression [25]. In pathologic circumstances, the cumulative evidence demonstrates that RCAN1 levels are altered in Down syndrome, Alzheimer's disease, Huntington disease, ischemic stroke, and spinal cord injuries [13-18,26]. Except for Huntington disease, RCAN1 has been shown to increase in most brain diseases. Consistent with that notion, we demonstrated upregulated RCAN1 expression in mouse hippocampi after acute seizures. Interestingly, the RCAN1 expression pattern after acute seizures differed between neurons and glial cells. Whereas RCAN1 was enhanced in the hippocam- 

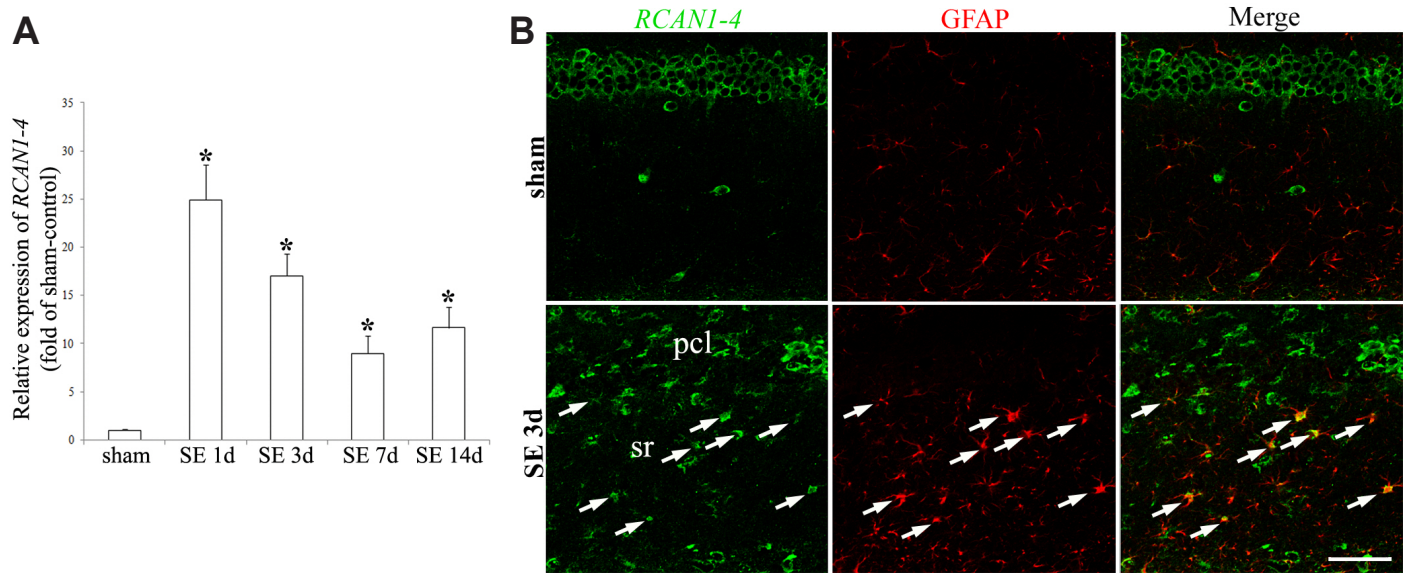

Fig. 4. Regulator of calcineurin 1 isoform 4 (RCAN1-4) mRNA expression in the hippocampus after pilocarpine-induced status epilepticus (SE). A qRT-PCR analysis showed that temporal RCAN1-4 transcription increased significantly in the hippocampus after SE, compared with sham-manipulated animals (A). Data presented are the mean \pm standard error of the mean, with ${ }^{*} p<0.05$ by one-way ANOVA followed by Duncan's post-hoc test. In situ hybridization showed that RCAN1-4 mRNA in the sham-manipulated animals was expressed in the pyramidal neurons of the hippocampus, whereas 3 day after SE, RCAN1-4 signals were detected in GFAP-positive astrocytes (arrows), along with pyramidal neurons (B). Scale bar in (B) $=50$ $\mu \mathrm{m}$. GFAP, glial fibrillary acidic protein; $\mathrm{pcl}$, pyramidal cell layer; sr, stratum radiatum.

pal dendritic regions from the acute phases of epileptogenesis until the time when seizure-induced neuronal damage occurred, glial RCAN1 emerged in the entire hippocampus at the subacute and chronic phases of epileptogenesis, suggesting differential functions of RCAN1 in epilepsy. To the best of our knowledge, this is the first report showing the comprehensive spatiotemporal expression pattern of RCAN1 throughout the epileptogenesis of an animal model of TLE.

Our immunohistochemistry and double immunofluorescence analyses indicate that RCAN1 induction in small cells with stellate morphology after acute seizures occurred in reactive astrocytes but not in microglia. Under physiologic conditions, RCAN1 expression was not detected in glial cells, which was consistent with the data from our sham-manipulated controls [27]. These results fit nicely with our previous report showing RCAN1 upregulation in GFAP-positive astrocytes after ischemic stroke, in addition to other researchers' results $[17,18]$. Moreover, calcium ionophore treatment markedly increased RCAN1-4 expression in primary astrocyte cultures, supporting the escalation of RCAN1 in astrocytes [28]. This was further corroborated by a report showing that the RCAN1-4 promoter was highly activated in the C6 glial cell line [12]. On the contrary, RCAN1 expression in Alzheimer's disease was detected only in neurons, without significant expression in astrocytes or microglial cells, suggesting a context-dependent cell type-specific role for RCAN1 [14,27]. Stroke and seizure activity can cause abrupt cellular and molecular changes, whereas Alzheimer's disease features a slow accumulative disruption of cell signaling; thus, it will be interesting to elucidate how astrocytic RCAN1 production is differentially stimulated upon different brain insults.

Among the three predominant RCAN1 isoforms expressed in the brain, it is well-known that RCAN1-4 can be induced by an intracellular calcium increase $[7,12,28,29]$. Although its basal expression levels are lower than those of RCAN1-1 in the brain [4], RCAN1-4 can be immediately upregulated in response to oxidative stress [20]. When we assessed the temporal expression pattern of RCAN1-4 mRNA after SE, we found a rapid hippocampal increase in RCAN1-4 transcription that was maintained for the following 2 weeks after acute seizures. Because epileptic seizures can cause catastrophic calcium influxes into the intracellular space, leading to excessive excitotoxic neuronal damage [21], enhanced RCAN1-4 transcription after prolonged seizures activity is plausible. Moreover, given that calcium overload is a common phenomenon in multiple types of brain injury, increased RCAN14 in ischemic stroke nicely supports our findings $[17,18]$.

Although the mechanisms of RCAN1 upregulation after acute seizures remain unclear, we propose a few possible explanations. In the early phases of epileptogenesis (before the occurrence of hippocampal cell death), neuronal RCAN1 was notably increased in our results. Because RCAN1-overepxressing neurons exhibit reduced viability upon oxidative stress [30] and prolonged seizure activity can generate explosive reactive oxygen species (ROS) [31,32], increased RCAN1 expression after SE could contribute to the delayed hippocampal neuronal deaths. This was further supported by evidence indicating that RCAN1-depleted cells displayed increased resistance to $\mathrm{H}_{2} \mathrm{O}_{2}$ stress [20]. The underlying mechanisms are thought to be related to RCAN1-triggered mitochondrial dysfunction and caspase activation, which results in more robust mitochondrial ROS and an increased susceptibility to oxidative stress-induced apoptosis $[12,30,33]$. However, contradictory reports claim that RCAN1 has a neuroprotective effect $[18,34]$. Specifically, RCAN1-1L overexpression could inhibit apoptosis under hypoxia [34], and RCAN1 knockout increased the infarct volume after ischemic 
stroke [18]. Therefore, more elaborate studies are needed on the role of RCAN1 in neuronal survival. With regard to glial RCAN1 expression in the subacute and chronic phases of epileptogenesis, we propose that RCAN1 is responsible for anti-inflammatory action. RCAN1 has been demonstrated to alleviate the production of diverse inflammatory cytokines and key inflammatory enzymes by modulating nuclear factor of activated T cell (NFAT) or nuclear factor kappa light chain enhancer of activated B cell (NF$\mathrm{kB})$ pathways $[8,10,17,18,28,35,36]$. Regardless of the involvement of calcineurin, a well-known RCAN1-interacting phosphatase, RCAN1 can promote NFAT activation and block NF-kB activity $[17,18,28,35]$. Because glial release of inflammatory mediators after acute seizures and prolonged enhancement of inflammation is a critical mechanism in epileptogenesis [37,38], RCAN1 induction in reactive astrocytes after SE could contribute to the attenuation of seizure-induced inflammation. The multiple functions of RCAN1 in brain injuries mean that further studies are required to understand all the roles of RCAN1 in epilepsy.

In summary, we have provided the spatiotemporal expression pattern of RCAN1 and its isoform RCAN1-4 in the hippocampus after pilocarpine-induced SE. We have also shown that the main cell type for the induced RCAN1 protein and RCAN1-4 mRNA after acute seizures is reactive astrocytes. Taken together, our results provide a scientific basis for the involvement of RCAN1 in epileptic seizures and the development of epilepsy.

\section{ACKNOWLEDGEMENTS}

This work was supported by National Research Foundation of Korea (NRF) grants funded by the Korean government (NRF2017R1A2B4002704 to S.Y.K. and NRF-2019R1A2C1003958 to K.-O.C.).

\section{CONFLICTS OF INTEREST}

The authors declare no conflicts of interest.

\section{REFERENCES}

1. Fisher RS, Acevedo C, Arzimanoglou A, Bogacz A, Cross JH, Elger CE, Engel J Jr, Forsgren L, French JA, Glynn M, Hesdorffer DC, Lee BI, Mathern GW, Moshé SL, Perucca E, Scheffer IE, Tomson T, Watanabe M, Wiebe S. ILAE official report: a practical clinical definition of epilepsy. Epilepsia. 2014;55:475-482.

2. Blair RD. Temporal lobe epilepsy semiology. Epilepsy Res Treat. 2012;2012:751510.

3. Becker AJ. Review: animal models of acquired epilepsy: insights into mechanisms of human epileptogenesis. Neuropathol Appl Neurobiol. 2018;44:112-129.

4. Hoeffer CA, Dey A, Sachan N, Wong H, Patterson RJ, Shelton JM,
Richardson JA, Klann E, Rothermel BA. The Down syndrome critical region protein RCAN1 regulates long-term potentiation and memory via inhibition of phosphatase signaling. J Neurosci. 2007;27:13161-13172.

5. Porta S, Martí E, de la Luna S, Arbonés ML. Differential expression of members of the RCAN family of calcineurin regulators suggests selective functions for these proteins in the brain. Eur J Neurosci. 2007;26:1213-1226.

6. Davies KJ, Ermak G, Rothermel BA, Pritchard M, Heitman J, Ahnn J, Henrique-Silva F, Crawford D, Canaider S, Strippoli P, Carinci P, Min KT, Fox DS, Cunningham KW, Bassel-Duby R, Olson EN, Zhang Z, Williams RS, Gerber HP, Pérez-Riba M, et al. Renaming the DSCR1/Adapt78 gene family as RCAN: regulators of calcineurin. FASEB J. 2007;21:3023-3028.

7. Cano E, Canellada A, Minami T, Iglesias T, Redondo JM. Depolarization of neural cells induces transcription of the Down syndrome critical region 1 isoform 4 via a calcineurin/nuclear factor of activated T cells-dependent pathway. J Biol Chem. 2005;280:29435-29443.

8. Minami T, Horiuchi K, Miura M, Abid MR, Takabe W, Noguchi N, Kohro T, Ge X, Aburatani H, Hamakubo T, Kodama T, Aird WC. Vascular endothelial growth factor- and thrombin-induced termination factor, Down syndrome critical region-1, attenuates endothelial cell proliferation and angiogenesis. J Biol Chem. 2004;279:5053750554.

9. Yang J, Rothermel B, Vega RB, Frey N, McKinsey TA, Olson EN, Bassel-Duby R, Williams RS. Independent signals control expression of the calcineurin inhibitory proteins MCIP1 and MCIP2 in striated muscles. Circ Res. 2000;87:E61-E68.

10. Hesser BA, Liang XH, Camenisch G, Yang S, Lewin DA, Scheller R, Ferrara N, Gerber HP. Down syndrome critical region protein 1 (DSCR1), a novel VEGF target gene that regulates expression of inflammatory markers on activated endothelial cells. Blood. 2004;104: 149-158.

11. Wu Y, Song W. Regulation of RCAN1 translation and its role in oxidative stress-induced apoptosis. FASEB J. 2013;27:208-221.

12. Sun X, Wu Y, Herculano B, Song W. RCAN1 overexpression exacerbates calcium overloading-induced neuronal apoptosis. PLoS One. 2014;9:e95471.

13. Fuentes JJ, Genescà L, Kingsbury TJ, Cunningham KW, Pérez-Riba M, Estivill X, de la Luna S. DSCR1, overexpressed in Down syndrome, is an inhibitor of calcineurin-mediated signaling pathways. Hum Mol Genet. 2000;9:1681-1690.

14. Harris CD, Ermak G, Davies KJ. RCAN1-1L is overexpressed in neurons of Alzheimer's disease patients. FEBS J. 2007;274:1715-1724.

15. Wong H, Levenga J, Cain P, Rothermel B, Klann E, Hoeffer C. RCAN1 overexpression promotes age-dependent mitochondrial dysregulation related to neurodegeneration in Alzheimer's disease. Acta Neuropathol. 2015;130:829-843.

16. Ermak G, Hench KJ, Chang KT, Sachdev S, Davies KJ. Regulator of calcineurin (RCAN1-1L) is deficient in Huntington disease and protective against mutant huntingtin toxicity in vitro. J Biol Chem. 2009;284:11845-11853.

17. Cho KO, Kim YS, Cho YJ, Kim SY. Upregulation of DSCR1 (RCAN1 or Adapt78) in the peri-infarct cortex after experimental stroke. Exp Neurol. 2008;212:85-92.

18. Sobrado M, Ramirez BG, Neria F, Lizasoain I, Arbones ML, Minami T, Redondo JM, Moro MA, Cano E. Regulator of calcineurin 1 
(Rcan1) has a protective role in brain ischemia/reperfusion injury. $J$ Neuroinflammation. 2012;9:48.

19. Lin HY, Michtalik HJ, Zhang S, Andersen TT, Van Riper DA, Davies KK, Ermak G, Petti LM, Nachod S, Narayan AV, Bhatt N, Crawford DR. Oxidative and calcium stress regulate DSCR1 (Adapt78/ MCIP1) protein. Free Radic Biol Med. 2003;35:528-539.

20. Porta S, Serra SA, Huch M, Valverde MA, Llorens F, Estivill X, Arbonés ML, Martí E. RCAN1 (DSCR1) increases neuronal susceptibility to oxidative stress: a potential pathogenic process in neurodegeneration. Hum Mol Genet. 2007;16:1039-1050.

21. Delorenzo RJ, Sun DA, Deshpande LS. Cellular mechanisms underlying acquired epilepsy: the calcium hypothesis of the induction and maintainance of epilepsy. Pharmacol Ther. 2005;105:229-266.

22. Jeong KH, Lee KE, Kim SY, Cho KO. Upregulation of Krüppel-like factor 6 in the mouse hippocampus after pilocarpine-induced status epilepticus. Neuroscience. 2011;186:170-178.

23. Racine RJ. Modification of seizure activity by electrical stimulation. II. Motor seizure. Electroencephalogr Clin Neurophysiol. 1972;32: 281-294.

24. Jang HJ, Kim JE, Jeong KH, Lim SC, Kim SY, Cho KO. The Neuroprotective effect of Hericium erinaceus extracts in mouse hippocampus after pilocarpine-induced status epilepticus. Int J Mol Sci. 2019;20:E859.

25. Peiris H, Keating DJ. The neuronal and endocrine roles of RCAN1 in health and disease. Clin Exp Pharmacol Physiol. 2018;45:377-383.

26. Wang G, Zhao Y, Liu S, Jia J, Lu T. Critical role of regulator of calcineurin 1 in spinal cord injury. J Physiol Biochem. 2016;72:605-613.

27. Ermak G, Morgan TE, Davies KJ. Chronic overexpression of the calcineurin inhibitory gene DSCR1 (Adapt78) is associated with Alzheimer's disease. J Biol Chem. 2001;276:38787-38794.

28. Canellada A, Ramirez BG, Minami T, Redondo JM, Cano E. Calcium/calcineurin signaling in primary cortical astrocyte cultures: Rcan1-4 and cyclooxygenase-2 as NFAT target genes. Glia. 2008; 56:709-722.
29. Hirakawa Y, Nary LJ, Medh RD. Glucocorticoid evoked upregulation of RCAN1-1 in human leukemic CEM cells susceptible to apoptosis. J Mol Signal. 2009;4:6.

30. Peiris H, Dubach D, Jessup CF, Unterweger P, Raghupathi R, Muyderman H, Zanin MP, Mackenzie K, Pritchard MA, Keating DJ. RCAN1 regulates mitochondrial function and increases susceptibility to oxidative stress in mammalian cells. Oxid Med Cell Longev. 2014;2014:520316.

31. Pauletti A, Terrone G, Shekh-Ahmad T, Salamone A, Ravizza T, Rizzi M, Pastore A, Pascente R, Liang LP, Villa BR, Balosso S, Abramov AY, van Vliet EA, Del Giudice E, Aronica E, Patel M, Walker MC, Vezzani A. Targeting oxidative stress improves disease outcomes in a rat model of acquired epilepsy. Brain. 2019;142:e39.

32. Shin EJ, Jeong JH, Chung YH, Kim WK, Ko KH, Bach JH, Hong JS, Yoneda Y, Kim HC. Role of oxidative stress in epileptic seizures. Neurochem Int. 2011;59:122-137.

33. Sun X, Wu Y, Chen B, Zhang Z, Zhou W, Tong Y, Yuan J, Xia K, Gronemeyer H, Flavell RA, Song W. Regulator of calcineurin 1 (RCAN1) facilitates neuronal apoptosis through caspase-3 activation. J Biol Chem. 2011;286:9049-9062.

34. Sun L, Hao Y, An R, Li H, Xi C, Shen G. Overexpression of Rcan1$1 \mathrm{~L}$ inhibits hypoxia-induced cell apoptosis through induction of mitophagy. Mol Cells. 2014;37:785-794.

35. Kim YS, Cho KO, Lee HJ, Kim SY, Sato Y, Cho YJ. Down syndrome candidate region 1 increases the stability of the IkappaBalpha protein: implications for its anti-inflammatory effects. J Biol Chem. 2006;281:39051-39061.

36. Junkins RD, MacNeil AJ, Wu Z, McCormick C, Lin TJ. Regulator of calcineurin 1 suppresses inflammation during respiratory tract infections. J Immunol. 2013;190:5178-5186.

37. Rana A, Musto AE. The role of inflammation in the development of epilepsy. J Neuroinflammation. 2018;15:144.

38. Vezzani A, French J, Bartfai T, Baram TZ. The role of inflammation in epilepsy. Nat Rev Neurol. 2011;7:31-40. 